\title{
Artikel
}

Gerd Schwerhoff*

\section{Das Pasquill im frühneuzeitlichen Deutschland. Ein Kommunikations- medium zwischen Schmähung und Kritik}


Abstract: The text deals with the genre 'pasquill' from the 16th to the 18th century in the German speaking world. Two strands of tradition can be ideally distinguished, which only gradually merge with each other. Originally, as in other regions of Europe, the Roman figure of the shoemaker Pasquino is adapted, who comments on actual politics or famous persons in mocking, more or less literary dialogues. This figure appears in printed works from the middle of the 16th century, mostly written by Protestants. At about the same time, the term 'pasquill' began to become synonymous with the mostly handwritten, anonymous libel, which is now increasingly criminalized by the authorities. The article characterises the early modern pasquill as a very special medium of communication, which served not only for personal defamation but also for objective criticism.

Keywords: Reformation, Aufklärung, Anwesenheitsgesellschaft, Ehre, Schmähschrift - reformation, enlightenment, face-to-face society, honour, libel

*Prof. Dr. Gerd Schwerhoff, TU Dresden, Institut für Geschichte, Professur für Geschichte der Frühen Neuzeit, gerd. schwerhoff@tu-dresden.de

\section{Erste Annäherungen}

Auf den ersten Blick scheint die Sache klar. "Pasquill", so definiert Gottsched 1758, „ist eine ehrenrührige Schrift, ohne Namen des Verfassers, aber mit dem Namen dessen, dem es gelten soll." ${ }^{1}$ Es handele sich um "Schmähschriften, darinnen der gute Namen, und die Sitten eines ehrlichen Mannes angetastet" werde. Deren Urheber wird folglich als "Pasquillant" bzw. als "Lästerer" qualifiziert - kein Ruhmestitel, wie Gottsched deutlich macht, denn Pasquille seien "in allen wohlbestellten Staaten verbothen", kein rechtschaffener Mann würde derartige Schriften verfertigen. "Ein solcher Lästerer schimpfet mehr sich, als den anderen. Seine eigenen Schmiralien schänden ihn, als ihren Urheber". Ein hauptsächliches Anliegen ist dem Gelehrten und Schriftsteller Gottsched die Grenzbefestigung zur sachlichen Auseinandersetzung:

1 Gottsched (1758) Beobachtungen, S. 229f.; dort auch alle folgenden Zitate. Der Text entstand im Teilprojekt G des SFB 1285. Vielen Dank vor allem an Jan Siegemund für seine Hinweise, die den Aufsatz wesentlich verbessert haben. Gedankt sei ebenfalls Stefan Beckert, Alexander Kästner, Max Rose, Wiebke Voigt sowie den Herausgeberinnen und dem Herausgeber für ihre Anregungen.
Man muß aber Streitschriften nicht mit Pasquillen verwechseln. In den ersten streite man um Wahrheiten, Geschichte, gelehrte Meinungen oder Lehrpuncte: in den andern aber geht es über die Personen her. Man kann in Meynungen uneins, und doch der Gegner Freund seyn. Nur ungezogene grobe Leute greifen die Personen ihrer Gegner an. Pasquille beschimpfen die schönen Wissenschaften [...] überhaupt.

Gottscheds kurze Einlassung zum Pasquill ist in mehrfacher Hinsicht interessant. Zum einen zeigt sie, wie selbstverständlich der Begriff Mitte des 18. Jahrhunderts als ein, deutsches Wort' eingemeindet wurde. Zum anderen benennt er mit der Anonymität des Verfassers und dem herabsetzenden Ziel der Schrift zwei zentrale definitorische Merkmale eines Pasquills. Und er stellt die Streitschrift als deren archetypisches Gegenstück heraus, in dem es um die Sache und nicht um die Person gehe. Damit hebt Gottsched auf ein zentrales Kennzeichen aufklärerischen Selbstverständnisses ab - ein Merkmal, das freilich den Charakter eines normativen Ideals besa $ß$ und das keineswegs mit der Realität aufklärerischen Schrifttums verwechselt werden sollte. ${ }^{2}$

2 Vgl. Oesterle (1986) Streitschrift. 
Aufschlussreich ist zugleich das, was Gottscheds kurzer Artikel nicht enthält, nämlich Erläuterungen zum medialen Aggregatzustand des Pasquills und zur Herkunft des Namens. Für beide Fragen hilft Zedlers unvermeidliches Universal-Lexikon weiter. 1740 wird unter dem Stichwort ,Pasquill' auf die Lemmata ,Famosus Libellus' bzw. ,Libellus Famosus' verwiesen. "Famosus Libellus, ein Paßquill, ist eine solche Schrifft, welche von einem verborgenen Auctore animo infamandi ist ans Licht gegeben worden", so führt der erste Artikel ganz im Sinn von Gottsched aus. Hier ist von "einer angeschlagenen oder ausgestreuten Schrifft" bzw. einem "Zettel" die Rede, aber auch davon, dass unter die Gattung auch Gemälde und Bilder gehören, die "zur Ehren-Verletzung eines andern" verfertigt worden sind. ${ }^{3}$ Der zweite Artikel gibt dann einen Hinweis zur etymologischen Herleitung des Begriffs: „Pasquill wird es von einem zu Rom befindlichen Bilde Pasquino genannt, an welches die Schmäh-Schriften von denen Verfertigern derselben pflegen angehefftet zu werden." ${ }^{4}$ Näheren Aufschluss gibt der Eintrag ,Pasquino'. Die antike Statue sei einst dort gefunden worden, wo sich früher die Werkstatt eines Schuhmachers (oder Schneiders) mit Namen Pasquino befunden habe, der zu seinen Lebzeiten die Schwächen seiner Mitbürger spöttisch aufs Korn genommen habe. In der Statue, so hätten die Nachbarn gewitzelt, sei Pasquino wiederauferstanden und es habe sich der Brauch entwickelt, an die Statue "bey der Nacht satyrische Schriften" gegen bestimmte Personen zu hängen, die man nicht offen anzugreifen wage. ${ }^{5}$

Bereits dieses erste Schlaglicht hat eine Vielzahl von Begriffen und Phänomenen erhellt und damit die Heterogenität dessen, worum es hier gehen soll: Das Pasquill wird mit Schmähschrift und libellus famosus gleichgesetzt; es kann sich um einen Text oder ein Bild handeln; es kann als handschriftlicher Zettel daherkommen, jedoch auch als Druck; selbst die Wertung ist nicht so eindeutig, wie Gottsched nahelegt, bezieht man die von Wohlwollen und Wertschätzung getragenen Berichte über den Pasquino in Rom mit ein. Insofern muss die lakonische Feststellung des Pioniers der Pasquill-Forschung aus dem Jahr

3 Zedler Bd. 9 (1735), Sp. 209 f.

4 Zedler Bd. 17 (1738), Sp. 773.

5 Zedler Bd. 26 (1740), Sp. 1148.
1838, in der Reformationszeit habe sich neben dem Spott- und Schmählied eine eigene Schriftgattung entwickelt, "die man Pasquille nennt", mit einem deutlichen Fragezeichen versehen werden. ${ }^{6}$ Die bislang einzige Sammlung einschlägiger Texte, die Edition von Bauer über Pasquille in den Fuggerzeitungen, hält diese Eingrenzung denn auch für zu restriktiv und verwendet deshalb das Schlagwort - auch ausweislich des Untertitels - synonym zu "jegliche(r) Form von Spott- und Schmähgedichten"; folglich definiert er Pasquill als "eine mündlich oder schriftlich verbreitete sprachliche Form, die zeitgenössische Personen und/oder Ereignisse in kritischer, satirischer, ironischer, spöttischer Weise behandelt und die zur Verbreitung bestimmt ist."7 Tatsächlich enthält seine Quellensammlung viele mehr oder weniger kunstvoll gereimte Texte, aber auch solche, die auf andere Weise literarisch gestaltet sind, als Dialog etwa oder als Gebetsparodie. Oft, aber nicht immer, richten sich diese Texte gegen konkrete Personen, manchmal kommentieren sie auch allgemeiner die Zeitläufte oder berichten von einzelnen Ereignissen. So berichtet ein Pasquill das unngerische kriegswesen vor Erlaw betreffende über eine vom kaiserlichen Heer verlorene Schlacht des Jahres 1596; trotz des Titels, so Bauer, handele es sich nicht um ein Pasquill im engeren Sinne, sondern um ein Zeitungslied mit einer ausführlichen Schlachtenschilderung. ${ }^{8}$ Mit einer erheblichen Unschärfe im zeitgenössischen Begriffsgebrauch ist also zu rechnen. Die neuere Forschung hat dem, wie das Beispiel Bauers zeigt, durchaus Rechnung getragen. Die eherne Grenze zwischen Schmähung und Kritik, wie sie nicht nur Gottsched im 18. Jahrhundert zog, wurde allerdings kaum je in Frage gestellt. ${ }^{9}$ Für ein unbefangenere Annäherung an das, ,Pasquill' als ein Kommunikationsmedium eigener Art empfiehlt es sich jedoch, auch die Zeit vor der Aufklärung zu betrachten und zugleich stärker die Sphäre außerhalb der gelehrten Welt der literarisch-wissenschaftlichen Öffentlichkeit in den Blick zu nehmen.

6 Voigt (1838) Pasquille, S. 340.

7 Bauer (2008) Pasquille, S. 21. Vgl. zu den Fuggerzeitungen https://fuggerzeitungen.univie.ac.at/ (letzter Zugriff: 02.01.2020).

8 Bauer (2008) Pasquille, S. $195 f$.

9 Vgl. kurz Wolf (2003) Pasquill; zuletzt Grunert (2019) Streiten und Strafen. 


\section{Archivalische Probebohrungen - das Beispiel Köln}

Dem Historiker der frühneuzeitlichen Stadt sind Pasquille keineswegs fremd. Sie werden in der archivalischen Überlieferung durchaus häufiger erwähnt; erhalten sind sie dagegen wesentlich seltener, weil sie - ihres ehrverletzenden Inhalts wegen - eher unterdrückt, vielfach sogar öffentlich ostentativ (etwa durch den Scharfrichter) vernichtet wurden, um anzuzeigen, dass die Ehrverletzung von der angegriffenen Person auf den Autor zurückfiel. ${ }^{10}$ So ist es bereits ein Glücksfall, wenn wir Näheres über Umstände und Inhalte erfahren wie bei einem Vorfall in der Reichsstadt Köln Anfang März 1593. Wegen eines verdächtigen Schriftstücks, eines „paßquill[s] oder [einer] schmehe schrifft", wurde der Fassbinder und ehemalige Soldat Lambert Deckens, gebürtig aus Maastricht, in städtische Untersuchungshaft genommen. ${ }^{11}$ Vor den Deputierten des Rates versuchte er seinen Umgang mit dem Text demonstrativ harmlos darzustellen. Er habe ihn unter anderen Schriftstücken in einem ,Tresor' der Botenwitwe Enne Schmitz gefunden, die er von Kind an kenne und bei der er zur Herberge liege. Den Inhalt des Pasquills kennen wir nicht, nur in einem sind sich Deckens und die befragten Zeugen einig: „e $\mathrm{e}$ ist vom auflauf de $\beta$ jahrs 13". Die Schrift handelte also von der innerstädtischen Unruhe der Jahre 1512/3, einem markanten Ereignis der Kölner Stadtgeschichte, das mit der Hinrichtung einiger führender Stadtpolitiker und der Ausarbeitung des sog. Transfixbriefes geendet hatte; mit diesem Dokument waren die Partizipationsrechte der Bürgergemeinde gegenüber dem städtischen Magistrat gestärkt worden. ${ }^{12}$ Das Pasquill war wohl recht umfangreich, denn die Leser mussten darin hin- und herblättern, und es war "auf ein liedtz weiß gedichtet". Wörtlich kennen wir lediglich die letzten Zeilen, die immerhin auch eine Idee von der Reimqualität vermitteln: „wer diesen brieff gedichtet, der ist lieber zu

10 Rublack (1995) Anschläge, S. 384; Bellingradt (2011) Flugpublizistik, S. 67f.; Grunert (2019) Streiten und Strafen, S. 175.

11 HAStK Bestand 35, Verfassung und Verwaltung G 228, fol. 119r-123r; dort alle folgenden Zitate. Vgl. dazu bereits Schwerhoff (2004) Öffentliche Räume, S. $131 f$.

12 Historisches Archiv der Stadt Köln (1996) Stadtrat, S. 85-92.
Coln alß zu Mastricht". ${ }^{13}$ Die Deputierten zitieren diese Zeile mit Bedacht, denn sie verdächtigen den gebürtigen Maastrichter Deckens, selbst der Autor zu sein. Der weist den Verdacht weit von sich, will den Brief an sich genommen haben, weil er neugierig war und den Inhalt nicht ganz verstand. Ursprünglich wollte er inn seinem Prokurator, dem Rechtsgelehrten Dussel, zur Ansicht bringen; als er inn nicht angetroffen habe, habe er das Dokument einigen anderen Leuten gezeigt, darunter dem Burggrafen (Hausmeister und Wächter) auf dem Frankenturm, dem städtischen Untersuchungsgefängnis. Auch die anderen Zeugen wollen so recht nicht verstanden haben, worum es in dem Brief genau ging. "Was lest Ihr vom Herrn Lyskirchen", will die Witwe Enne nach eigenen Angaben zu Deckens gesagt haben, "der ist doch schon lange tot". Nebenbei gesagt, könnte diese Bemerkung darauf hindeuten, dass das Pasquill nicht allein von der innerstädtischen Unruhe des Jahre 1513 handelte, sondern auch von anderen Tumulten wie jenem von 1482, bei dem ein Angehöriger des alten Patriziergeschlechts von Lyskirchen eine Hauptrolle gespielt hatte; Werner von Lyskirchen hatte damals seine Opposition zum Rat mit dem Leben bezahlt. ${ }^{14}$ Enne sagte weiterhin aus, dass ihr Neffe, der Notar Johann Schmidt, die Schriftstücke samt des Pasquills vor drei Jahren in ihrem Tresor deponiert habe. Natürlich, und deswegen fragen die Ratsbeamten derartig genau nach, ist auch eine andere Interpretation des ganzen Vorganges möglich. Die Bürger mochten genau verstanden haben, was im Pasquill, das Lambert angeblich in die Finger gefallen war, stand und sie hatten ihn von Hand zu Hand wandern lassen, um sich daran zu ergötzen. Darauf deutet auch die Tatsache hin, dass die Schmähschrift nun auch auf dem Frankenturm für Wirbel sorgte. Denn dort, im Gefängnis, hatte der Gefangene Lambert Gelegenheit, sich mit anderen Häftlingen zu unterhalten. Darunter war auch ein gewisser Johann Kramer, ein ehemaliger städtischer Beamter, der wegen einer Korruptionsaffäre gefangen saß und sich bei den Ratsherren wegen seiner Aufsässigkeit bereits herzlich unbeliebt gemacht hatte. Dieser habe sich vernehmen lassen, so erzählt Lambert später in einem zweiten Verhör, er kenne noch etliche

13 HAStK Bestand 35, Verfassung und Verwaltung G 228, fol. 122 r.

14 Historisches Archiv der Stadt Köln (1996) Stadtrat, S. $83 f$. 
Briefe, die das Jahr 13 angingen; einmal hätte er jemandem ein Viertel Wein angeboten, um ihm einen solchen Brief zu kopieren. Auch wisse er, wo noch mehr dieser Briefe vorhanden seien. Schließlich habe Kramer in Gegenwart des Burggrafen die Frage aufgeworfen, "wan er dergleichen brieff einen bei sich hett, ob er darumb woll in pericull [Gefahr] kommen solte, wan er denselben nicht gedichtet". ${ }^{15}$ Diese Formulierung dürfte als kaum verklausulierte Kritik an einer Obrigkeit gemeint gewesen sein, die harmlose Stadtbewohner für ein Schriftstück inkriminierte, das diese nicht selbst verfasst hatten. Kramer blieb weiterhin gefangen. Was die Schmähschrift selbst anlangte, scheint der Rat aber endlich zum Schluss gekommen zu sein, dass die Affäre tatsächlich lediglich "alte historien" (wie es an einer Stelle heißt) betraf und keine aktuelle Kritik an der Obrigkeit beinhaltete. Lambert Deckens durfte nach rund drei Wochen den Turm wieder verlassen, allerdings nicht ohne die Ermahnung, sich zu mäßigen.

Schmähschriften und Pasquille prägten auch später innerstädtische Konflikte in der Reichstadt Köln. In besonderer Intensität war das in den bewegten Jahrzehnten vor und nach 1700 der Fall, wobei die Zeit des sog. Gülich-Aufstandes in den 1680er Jahren herausragt. ${ }^{16}$ Auch nach der Bestrafung der Aufständischen und dem Ende der Revolte entfalteten Schmähschriften weiterhin ihr Drohpotential. ${ }^{17}$ Nächtlich angeheftete Pasquille zeugen davon, dass der Unmut der Bürger gegen die etablierten Eliten anhielt. Zahlreiche Verbote des Rates und die symbolische Vernichtung der Schmähzettel durch den Scharfrichter am Pranger hatten nur begrenzten Erfolg. 1698 drang ein fernes Echo der Kölner Schmähkampagnen sogar bis nach Wien. ${ }^{18}$ Der ständige kaiserliche Gesandte Graf Schellardt berichtete Mitte Oktober, in den vergangenen Tagen seien heimlich Pasquillen in die Häuser verschiedener Bürgermeister geworfen worden; darin hätte sich, der gemeine Pöbel' über die Teuerung des Brotes beschwert und Abhilfe begehrt. Mehrere

15 HAStK Bestand 35, Verfassung und Verwaltung G 228, fol. 122 r.

16 Bellingradt (2011) Flugpublizistik, S. 67, 91 sowie S. 102-124.

17 Vgl. Schwerhoff (2017) Köln im Ancien Régime, S. 64-68 und S. 157f.

18 HHStA Wien, Bestand Reichskanzlei, Diplomatische Akten, Köln: Berichte 2a, S. 518-525. der Pasquille werden wörtlich zitiert und geben einen Eindruck davon, wie unterschiedlich das im Kern identische Anliegen vorgetragen wurde. Alle beginnen mit einer direkten Anrede (,Ihr Herrn Bürgermeister'), alle beschweren sich darüber, dass das Brot so teuer sei, dass der gemeine Mann es sich kaum leisten könne; und alle drohen den Herren, ,Gülichs Wesen' sei noch nicht vollbracht, mit anderen Worten: der Aufstand könne jederzeit wieder losbrechen. Zwei der Texte sparen nicht mit Schmähungen gegen die Bürgermeister ('Schelme', ,Kornwucherer'). Der dritte kommt weniger affektgeladen daher, droht aber dafür den Stadtherren kapitale Strafen an, wenn die Brotpreise nicht gesenkt würden - man wolle ihnen dann ,den Kopf hinter die Füße legen'.19 Der Effekt der Pasquille ist schwer einzuschätzen. Einerseits verstärkte die städtische Obrigkeit ihre ordnungspolizeilichen Bemühungen und Fahndungsanstrengungen, offenbar ohne dass sie an die Autoren herankam. ${ }^{20}$ Andererseits nahm sie derartige Protestsignale so ernst, dass sie ihre Versorgungspolitik zu verbessern suchte.

Die beiden aufgeführten Beispiele aus der Reichsstadt Köln liegen fast einhundert Jahre auseinander, und auch in formaler wie inhaltlicher Hinsicht gibt es gewichtige Unterschiede: Bei dem Pasquill, mit dem Deckens und Kramer umgingen, handelte es sich offensichtlich um ein - wenn auch nicht sehr kunstvoll - gereimtes Dokument; die späteren Pasquille kamen dagegen als eilig aufs Papier geworfene Drohworte daher. Das eine bezog sich auf ein historisches Ereignis, das schon Generationen zurücklag, das andere benutzte eine jüngere Unruhe als Drohkulisse und richtete sich konkret an die derzeitigen Amtsträger. Gemeinsam war innen aber eben auch die Referenz auf ein politisches Aufstands-

19 Eine Kostprobe, (HHStA Wien, Bestand Reichskanzlei, Diplomatische Akten, Köln: Berichte 2a, S. 519): „Ihr herrn burgermeister, die gemeint ist es baldt müeth, daß wir das brodt so thür bezahlen müßen und auch daß schelmen armenhau $ß$ underhalden mußen in der stadt daß der burger ihre nahrung abgenohmen wird, wan es mit dem brodt nit geändert wird und auch mit dem anderen niet, dan werde wirr bald daß gülichs wesen anfangen noch reger als zu befür, dan willen wir daß hauß aufbauen, und setzen den kopff an die bürgermeisters haußer. Ihr gaffel haußer machet ihr auch klahe, wan es angehet."

20 Z. B. HAStK Bestand 10, Ratsprotokolle Nr. 133, fol. 92v; Bestand 35 Verfassung und Verwaltung, G 268, fol. $65 a-66 b, 74 b-78 a, 95 b$. 
geschehen und damit die Tatsache, dass hier deutlich mehr zur Debatte stand als eine Animosität gegenüber konkreten Personen. Dass Köln hier keineswegs ein außergewöhnliches Beispiel darstellt, zeigt ein Blick auf andere Orte, etwa auf die Medienhochburg Hamburg zur gleichen Zeit. ${ }^{21}$

\section{Die rechtshistorische Perspektive}

Nimmt man den Begriff des Pasquills - wie es Gottsched tat - synonym für ,Schmähschrift', bzw. libellus famosus, stellt man damit also vollkommen auf den (Un-)Rechtscharakter der schriftlichen Äußerung als ehrverletzende Schmähung ab und nicht auf seine formale Gestalt, dann eröffnet sich eine sehr weit zurückreichende Perspektive: Bis in die Antike lässt sich mit der bis heute maßgebenden Arbeit von Schmidt die Androhung schwerster Strafen für die Anfertigung von derartigen Schmähschriften zurückverfolgen. ${ }^{22}$ Voreilig wäre es allerdings, von einer historischen Konstante zu sprechen. Denn es fällt auf, wie wenig einschlägige rechtliche Regelungen für das Mittelalter bekannt sind. Eine Ausnahme bildet hier das Landrecht des Schwabenspiegels, das im letzten Drittel des 13. Jahrhunderts denjenigen das Rädern androht, "die schreyben prieff oder haissent sy ander leut schreiben vnd seczend die selben dran mit iren namen vnd werffent die prief an die strass das sy die leut auf heben vnd lesen". ${ }^{23}$ Offen bleibt dabei, ob sich dieser Passus inhaltlich direkt auf den vorangehenden bezieht, wo die nämliche Sanktion für Verleumder angedroht wird, die andere als Ketzer und Sodomiter bezeichnen. Auch eine Sichtung der städtischen Statuten des späteren Mittelalters erbringt keinen üppigen Ertrag. Zu den wenigen Belegen gehört das Braunschweiger Stadtrecht aus der ersten Hälfte des 14. Jahrhunderts, in dem das Schreiben, Verteilen oder Anheften von Briefen verboten wird, die den Ruf eines anderen beschädigen; der Täter solle für einen, unrechten Mann' gehal-

21 Bellingradt (2011) Flugpublizistik, S. 138 u.ö. 22 Schmidt (1985) Libelli Famosi, S. 72f. Pars pro toto für die neuere kulturhistorische Forschung Fuchs (1999) Ehre, besonders S. 153-168.

23 Eckardt (1974) Schwabenspiegel Landrecht 174a, S. 253; vgl. Schmidt (1985) Libelli Famosi, S. $226 f$. ten werden. Ähnlich sieht das Berliner Stadtrecht vor, dass als Verräter gerichtet werden sollte, wer ohne Einwilligung des Rates heimlich Briefe an den Pranger hänge. ${ }^{24}$

Dichter werden die Belege erst mit dem 16. Jahrhundert. Die Bamberger Halsgerichtsordnung von 1507 (Bambergensis) bzw. vor allem die Kaiserliche Halsgerichtsordnung von 1532 (Carolina) markieren hier eine neue Stufe. Sie regeln in fast gleichlautenden Worten die "Straff Schrifftlicher unrechtlicher peinlicher Schmehung": Wer jemandem, so heißt es in Artikel 110 der Carolina, "durch schmachschrifft zu latein libel famoß genant" unrechtmäßig "laster und übel" zumisst, soll mit derselben Strafe sanktioniert werden, die auf die Beschuldigung eigentlich ausgesetzt ist. ${ }^{25}$ In den späteren Kommentaren zu diesem Artikel wird der Tatbestand einer anonymen Äußerung "ad infamiam alicuius in publico loco" weiter geschärft. ${ }^{26}$ Noch im einschlägigen Kapitel "Von der schmehelichen schrifft. De Libello Famoso" des sächsischen Rechtshandbuchs von Kilian König (gestorben 1526) ) $^{27}$ sucht man allerdings den Begriff ,Pasquill' vergebens. Das änderte sich erst im Zuge einer aufblühenden Polizeigesetzgebung im Reich, die sich zunehmend mit Schmähschriften befasste. Eine deutliche Zäsur bildet hier die Reichspolizeiordnung von 1548, die einen eigenen Abschnitt "Von Schmäh-Schrifften, Gemählden, und Gemaechten" enthält. Dort erfahren die Bestimmungen gegen die libelli famosi eine neue und verstärkte Akzentuierung dadurch, dass sie in den Kontext der Zensur von Druckerzeugnissen gerückt werden. Ausdrücklich zum Schutz der katholischen Lehre wird dort u.a. "schmähliches, pasquillisches [sic!] oder ander weiß wie den Namen haben möchte" verboten, egal ob es "gedicht, geschrieben, in Druck gebracht, gemahlt, geschnitzt, gegossen oder gemacht" sei. In leicht modifizierten Worten wendet sich auch die Reichspolizeiordnung des Jahres 1577 gegen "Famos-Bücher oder Schrifften, es

24 Schmidt (1985) Libelli Famosi, S. 228. Zum Verbot des Absingens von Schandliedern oder des Dichtens von Schmähversen vgl. ebd. S. 230-233.

25 Schmidt (1985) Libelli Famosi, S. $236 f$.

26 Schmidt (1985) Libelli Famosi, S. 244 (so Benedict Carpzov in seiner Practica rerum criminalium von 1635). Vgl. insgesamt auch Buehler (2015) Defamation, S. 32ff.

27 König (1541) Processus, fol. LXVIv. Vgl. dazu den Aufsatz von Siegemund (2020) Schmähschriftprozess. 
habe der Author seinen Namen darunter gesetzt, oder nit". ${ }^{28}$ Dass hier das Kriterium der Anonymität ausdrücklich für irrelevant erklärt wird, unterstreicht die Offenheit der Kategorie ,Schmähschrift' und das Interesse der Obrigkeit an einer möglichst flexiblen Regelung.

Die Reichspolizeiordnung von 1577 verbietet im Übrigen im gleichen Abschnitt eine weitere Praxis, die Parallelen zum libellus famosus aufweist, von diesem aber kategorial eindeutig zu unterscheiden ist: den Brauch nämlich, dass ein Gläubiger, der vom Schuldner oder dessen Bürgen nicht bezahlt wird, „dieselbigen mit schändlichen Gemähld und Brieffen, öffentlich anschlagen, schelten, beschreyen und beruffen lässt". ${ }^{29}$ Der Passus zielt auf jene Scheltbriefe und Schandgemälde, die ab dem zweiten Drittel des 14. Jahrhunderts einsetzen und die bis ca. 1600 verwendet werden. Durch die entehrende Darstellung des Gegners bzw. seiner Symbole in Verbindung mit unreinen Tieren oder mit Instrumenten des Strafvollzuges soll dieser zur Begleichung der Schuld gezwungen werden. ${ }^{30} \mathrm{Im}$ Unterschied zum anonymen Schmähbrief besteht allerdings in diesem Fall kein Zweifel über den Urheber des Dokuments, eben den Gläubiger, der mittels des Briefes bzw. des Bildes auf dem Wege der Selbsthilfe und unter Umgehung der Gerichte sein Gegenüber zur Begleichung der Schuld zu nötigen sucht. Dass diese Tradition prinzipiell in andere Kontexte übertragen werden konnte, zeigt jene Karikatur des lutheranischen Pastors Simon Haderdey, die 1605 im bikonfessionellen Augsburg ein 20-jähriger Student des Jesuitenkollegs zeichnete. Sie zeigt ihn sehr lebensecht, wie er in der Hölle von Dämonen gequält wird. Bereitwillig gestand der junge Mann, dass er dergleichen öfter fertige und sich damit unter Gesinnungsgenossen ein Zubrot verdiene. ${ }^{31}$

Nicht nur dieses Beispiel zeigt, dass das Genre der Schmähbriefe weder formal noch medial näher bestimmt war: Es konnte geschrieben oder gemalt, gereimt oder ungereimt, handschriftlich

28 Reichspolizeiordnung von 1548 Art. 34, nach Senckenberg (1747), S.604f.; Reichspolizeiordnung 1577, Art. 35, nach Senckenberg (1747), S. 396.

29 Reichspolizeiordnung 1577, Art. 35, nach Senckenberg (1747), S. 397.

30 Vgl. umfassend Lentz (2004) Konflikt, Ehre, Ordnung. 31 Creasman (2012) Censorship, S. 134-136 (mit Abbildung). oder gedruckt daherkommen; entscheidend war seine auf die Ehrverletzung eines Gegners gerichtete Funktion. Trotz dieser Unbestimmtheit deutet alles darauf hin, dass die typische Schmähschrift handschriftlich verfasst wurde - kaum verwunderlich, denn wer anonym eine ehrverletzende Beschuldigung gegen einen Kontrahenten oder gegen die Obrigkeit ausstreuen wollte, dürfte kaum den Zugang zum aufwendigen und vergleichsweise gut zu kontrollierenden Druckverfahren gesucht haben. Einfacher war es, einen Fetzen beschriebenen oder gezeichneten Papiers an einem zentralen Ort der Stadt zu deponieren und darauf zu vertrauen, dass er gefunden, vorgelesen und sein Inhalt weiter kommuniziert wurde. Insofern bestand eine enge Verbindung zur kommunikativen Gattung des mündlich weitergetragenen Gerüchts. ${ }^{32}$ Dass die antike Tradition der Kriminalisierung von libelli famosi seit dem Beginn des 16. Jahrhunderts wiederbelebt und ausgebaut wurde, ist aber wohl trotzdem im größeren Kontext obrigkeitlicher Bemühungen zu verstehen, die Erzeugnisse des Druckmarktes besser zu kontrollieren, der sich im Zuge der Reformation gerade stürmisch entfaltete. ${ }^{33}$

\section{Die (literarische) Tradition des Pasquino}

Mit dem Beginn des 16. Jahrhunderts betritt auch jene Figur des Pasquino die öffentliche Bühne, die sich später partiell mit der älteren Tradition des libellus famosus verbinden sollte. Die spezifische Pasquino-Tradition darf als ein international vergleichsweise gut erforschtes Feld gelten, in dem bislang vor allem literaturwissenschaftliche Interessen bestimmend waren. $^{34}$ Die tatsächliche Herleitung des Namens ,Pasquino' wird sich wohl kaum mit letzter Sicherheit aufklären lassen. Die schon in Zedlers Lexikon kolportierte, weit verbreitete Erzählung über den scharfzüngigen Handwerker, dessen Namen dann auf die in der Nachbarschaft ausgegrabene antike Statue übertragen worden sei, konkurriert mit anderen,

32 Holenstein/Schindler (1992) Geschwätzgeschichte(n); Fenster/Smail (2003) Fama.

33 Vgl. Buehler (2015) Defamation.

34 Kurz Wolf (2003) Pasquill, Sp. 682f.; Romano (2006) La satira. 
metaphorischen Deutungen. ${ }^{35}$ Unumstritten ist jedenfalls, dass der später mit diesem Namen bezeichnete Torso 1501 ausgegraben, von Kardinal Oliviero Carafa an einer Ecke seines Palastes aufgestellt und durch eine neue Inschrift gekennzeichnet wurde. ${ }^{36}$ Schon Mitte August des gleichen Jahres erwähnt ein Tagebuch Schmähverse gegen den Borgia-Papst Alexander VI. auf einem Zettel ("cedula"), der an der "statue magistri Pasquino" angebracht gewesen sei; derartige Zettel seien überdies in der ganzen Stadt verteilt gewesen. ${ }^{37}$ Diese schnelle Abfolge könnte darauf hindeuten, dass es sich um eine neue Manifestation von schon länger bestehenden humanistischen Gebräuchen gehandelt haben könnte. Denn die 'Pasquinate', die satirischen Epigramme, Sonette und Stanzen, die in der folgenden Zeit produziert wurden und die rituellen Festivitäten der ,Pasquinaden', die am Markusfest und zu anderen Gelegenheiten unter Einbeziehung der Statue in Szene gesetzt wurden, waren keineswegs Produkte der Kultur des, einfachen Volkes', sondern prominente Ereignisse, die unter dem Patronat von Carafa und anderen Kardinälen stattfanden. Erstmals 1509 wurden die "Carmina quae ad pasquillum fuerunt posita" zum Teil abgeschrieben und in Druck gegeben. ${ }^{38}$ Aus ordnungspolitischen Gründen wurde allerdings die offizielle Feier $a b$ 1519 verboten. Eine Unterdrückung der z.T. politisch subversiven und persönlich verletzenden Texte gelang letztlich nicht. Erhalten sind aus dem späteren 16. Jahrhundert vor allem gedruckte Pasquinaden, handschriftliche Überlieferung stellt dagegen den absoluten Ausnahmefall dar. ${ }^{39}$ Im Übrigen hat sich die Tradition des Pasquino, wenngleich nicht ohne Unterbrechungen, in der italienischen Hauptstadt bis heute erhalten. ${ }^{40}$

Schnell wurde sie zudem Ausgangspunkt für die Ausbreitung des Brauchs auch an anderen Orten. ${ }^{41}$ Zunächst , antworteten' andere römische

35 Reynolds (1985) Carafa, S. $189 \mathrm{f}$.

36 Reynolds (1985) Carafa, S. 181, Anm. 17.

37 Reynolds (1985) Carafa, S. 181, Anm. 16.

38 Reynolds (1985) Carafa, S. 185.

39 Burke (1986) Beleidigungen, S. 106 bzw. Anm. 19 auf S. 206.

$40 \mathrm{Vgl}$. Eintrag ,Pasquino'. Wikipedia: https://de.wikipedia.org/w/index.php?title $=$ Pasquino\&oldid $=186209496$ (letzter Zugriff: 02.01.2020).

$41 \mathrm{Zu}$ Folgenden vor allem vor allem Lastraioli (2003) „Pasquini"; dies. (2010) Pétromarchies.
Statuen auf die spitzen Bemerkungen Pasquinos und produzierten weitere Spottverse, zuerst diejenige des Flussgottes Marforio. Doch entfaltete sich auch über Rom hinaus, in anderen italienischen Städten wie Florenz oder Venedig, eine Tradition der, sprechenden Steine'. Und es dauerte nicht lange, da begann der Begriff ,Pasquill ein literarisches bzw. druckmediales Eigenleben auch außerhalb der "Lästerschule Italien" (Burckhardt) zu entwickeln. ${ }^{42}$ So prägte der französische Dichter Joachim Du Bellay aufgrund seiner italienischen Erfahrungen den Begriff des Wettkampfs der Steine, der ,Petromachie'. ${ }^{43}$

Dieses Eigenleben allerdings wurde bislang vorwiegend für den romanischen Sprach- und Kulturkreis erforscht. Nachgewiesen sind überdies einige Dutzend von im niederländischen Raum gedruckten Flugschriften mit einem dezidierten Bezug auf die Pasquino-Figur, angefangen mit einer niederländischen Übersetzung von PasquilIus extaticus aus der Feder des Italieners Curione aus dem Jahr $1565 .{ }^{44}$ Umfang und Gestalt der literarischen Pasquino-Tradition im deutschsprachigen Mitteleuropa scheinen dagegen weitgehend unerforscht zu sein. ${ }^{45}$ Immerhin hatte bereits die fast zweihundert Jahre alte Materialsichtung von Johannes Voigt gezeigt, dass im Reich die Pasquino-Gestalt ebenfalls relativ schnell und dankbar aufgenommen wurde, natürlich vor allem von protestantischer Seite in papstkritischer Absicht. ${ }^{46}$ Eine systematische Sichtung, die zumindest in Bezug auf die gedruckten Quellen auf der Grundlage von VD 16 (Verzeichnis der im deutschen Sprachbereich erschienenen Drucke des 16. Jahrhunderts) nun besser denn je möglich ist, steht bislang allerdings aus.

Eine erste flüchtige Recherche zeigt, dass schon um 1520 in mehreren Ausgaben ein Büchlein mit dem Titel „Pascuillus" erschien; es sollte über die Listen der Römer bei der Schaffung vieler Kardinäle aufklären, die das Ziel verfolgten, alle Bistümer in Deutschland unter ihre Herrschaft zu bringen. Um dem Leser den Kontext

42 Zitat nach Fuchs (1999) Ehre, S. 161.

43 Lastraioli (2010) Pétromarchies, hier S. 233.

44 Dingemanse/Meijer Drees (2006) Pasquino, S. $484 f$.

$45 \mathrm{Vgl}$. dazu die sehr allgemeinen Bemerkungen ohne weitere Quellen- und Literaturverweise bei Lastraioli (2006) Pasquillus, S. 466.

46 Vgl. Voigt (1838) Pasquille, S. 337 u.ö.; weiterhin Schade (1863) Satiren, Bd. 3 Register cf. „Pasquillus". 
des Dialogs zwischen Pasquillus und Cirus zu erklären, wird dort eingangs die römische Tradition der Pasquino-Statue erklärt. ${ }^{47}$ Mag es sich bei diesem Text um Bearbeitungen italienischer Vorlagen handeln und noch nicht unbedingt um ein originäres Produkt der Reformationszeit, so verhält es sich bei späteren Varianten anders. Die "Unterredung zwischen Pasquill und einem Deutschen" über das - tatsächlich zunächst verschobene, dann abgesagte - Konzil zu Mantua aus dem Jahr 1537 ist als deftiger volkssprachlicher Dialog gestaltet, bei dem der Spötter den redlichen Fremden über die angeblichen, keineswegs ernst gemeinten Kirchenreformpläne des Papstes aufklärt und inn ermahnt, beim Evangelium zu bleiben. Holzschnittartig werden hier die redlichen Deutschen den bösen ,Romanisten' und ,Papisten' gegenübergestellt. Und wenn auch der Deutsche zunächst konstatiert, der scharfsinnige und scharfzüngige Pasquillus könne Rom nicht verlassen, weil die Stadt seines "vermanens und scheltens" nicht entbehren könne, so schließt sich der Spötter doch am Ende dem Deutschen an, um in der nächsten Wirtschaft zusammen mit dessen Landsleuten ein Bier zu nehmen - denn dort könne er reden, was er wolle. ${ }^{48}$ Konjunktur hatten schließlich Druckschriften mit dem Begriff ,Pasquillus' o.ä. im Titel im Umfeld des Schmalkaldischen Krieges, z.B. in Form eines Schmähgedichtes, in dem die Beratung zwischen dem Papst und seinem obersten Herren, dem Teufel, in Szene gesetzt wird. ${ }^{49}$ Wie groß die Bandbreite des Möglichen in jener Zeit war, demonstriert im Vergleich jener Pasquillus Germanicus In Quo Causa praesentis belli attingitur aus dem Jahr 1546, auf

47 Vgl. Pascuillus (1520), VD16 P 850; vgl. weitere Auflagen VD16 P 851-855.

$48 \mathrm{Vgl}$. EVANGELIA (1592), VD16 U 188; weiterhin auch Ein vnderredung (1537), VD16 U 187; zur satirischen Thematisierung des Konzils von Mantua ebenfalls Voigt (1838) Pasquille, S. 418-429.

49 Vgl. Pasquillus. Newe Zeyttung vom Teüffel (1546), VD16 S 3175; weitere Auflagen VD16 S 3176 - 3183 sowie ZV 13961; dazu Voigt (1838) Pasquille, S. 509-512. Für weitere Nachweise Waldeck (1910/11) Publizistik [II], S. 60 und S. 64-66, der allerdings nicht auf Gattungsfragen eingeht. Dem Gespräch zwischen einem verstorbenen Landsknecht und dem heiligen Petrus an der Himmelspforte, das etwa zur gleichen Zeit erschien (EJn Gesprech [1547], VD16 G 1882), wurde die Bezeichnung ,Pasquill' dagegen vom ersten Herausgeber aufgedrückt (Matthias: Ein Pasquill); vgl. allgemein Haug-Moritz (2002) "Geschwinde Welt". die Kaufmann aufmerksam gemacht hat: Hier steht die Titelfigur in keinerlei Verbindung zum tatsächlichen Inhalt der Schrift, wo verschiedene zeithistorische Akteure, der Papst und der Kaiser ebenso wie einzelne Reichsfürsten und Städte, "mit biblischen Worten vornehmlich prophetischer oder apokalyptischer Provenienz charakterisiert" werden. ${ }^{50}$ Eine nähere Untersuchung der gedruckten Pasquinaden im deutschsprachigen Raum Mitte des 16. Jahrhunderts muss zukünftigen Studien vorbehalten bleiben. Hier soll vorläufig die Feststellung genügen, dass die Verfasser des einschlägigen Artikels der Reichspolizeiordnung von 1548 höchstwahrscheinlich diese Drucke vor Augen gehabt haben werden, als sie das Verbot von "schmählichen" oder „pasquillischen" Schriften gegen die katholische Lehre verfassten. ${ }^{51}$

\section{Die Schmähschrift als ,Pasquill}

Am Beginn des 16. Jahrhunderts, so lässt sich die Geschichte des Pasquino bis hierher resümieren, entstand rund um eine antike Statue in Rom eine Tradition gelehrter Spottverse. Zumindest ursprünglich handelte es sich bei diesen Pasquinaden wohl um das gelehrte Spiel eines kleinen humanistischen Zirkels, selbst wenn es sich vor dem Forum der römischen Stadtöffentlichkeit vollzog. Medial beschränkte sich dieses Spiel am Anfang auf handschriftliche Zettel. Schnell aber wurden diese Zettel für den Druck adaptiert und die sprechenden Statuen vervielfältigten sich, zunächst in Rom, dann auch darüber hinaus. Die Figur des Spötters Pasquino wurde in verschiedenen gedruckten Dialogen quer durch Europa zum Sprachrohr öffentlicher Kritik. Das geschah auch im deutschen Sprachraum, insbesondere von Seiten der Protestanten. Dort firmierten die entsprechenden Schriften unter dem Titel ,Pasquill(us)'.

Längerfristig jedoch sollte jene andere Bedeutungsfacette des Begriffs dominieren, die sowohl in den Lexika des 18. Jahrhunderts als auch in der Kölner Alltagspraxis begegnet: seine Nutzung als Synonym für die (anonyme und hand-

50 Kaufmann (2006) Konfession und Kultur, S. 58 unter Bezug auf PASQVILLIVS GERMANICVS (1546), VD16 P 843. 51 Reichspolizeiordnung von 1548 Art. 34, nach Senckenberg (1747), S. 604f. 
schriftliche) ,Schmähschrift'. Diese Gleichsetzung erschien der Forschung meist selbstverständlich und wurde kaum je wirklich problematisiert. Versteht man die Gleichsetzung jedoch als erklärungsbedürftig, dann lassen sich einige grundsätzliche Beobachtungen treffen. Erstens gibt es eine programmatische Unschärfe des Terminus: Anders als bei den literarischen Pasquino-Texten, die sich in der Regel durch Dialogizität und Reimform auszeichnen, ist mit dem Begriff Pasquill für Schmähschriften - jedenfalls auf längere Sicht - keinerlei formale, mediale oder inhaltliche Präzisierung verbunden. Seit der Darstellung Voigts aus dem Jahr 1838 folgt die Charakterisierung der einschlägigen Texte eher einer pluralen Additionslogik („Über Pasquille, Spottlieder und Schmähschriften [...]"). Ähnlich macht es noch der neuere Handbucheintrag Kuhns über die heterogene Trias von ,ballads, libels, and songs'; das Pasquill wird dort lediglich indirekt angesprochen..$^{52}$ Für die jeweilige Obrigkeit dürfte eine lockere Definition dessen, was unter, Schmähschrift' zu verstehen war, ohnehin den Vorteil gehabt haben, ihr einen weiten Beurteilungs- und Handlungsspielraum zu bewahren. ${ }^{53}$

Zweitens scheint sich dieser weite Begriffsgebrauch besonders auf Mitteleuropa zu konzentrieren. Neben dem deutschen ist insbesondere der niederländische Sprachraum hervorzuheben, wo ,pasquil' bzw. ,pasquin' nicht nur die Bedeutung von libellus famosus bzw. Schmähschrift annahm, sondern ebenfalls - ähnlich wie im Deutschen das Substantiv „paskwillist" oder "paskwillant" für den Autor dieser Schmähtexte und das Verb "paskwilleeren" in Gebrauch kam. ${ }^{54}$ Umgekehrt war im frühneuzeitlichen England, Pasquil' zwar als Schutzpatron der Spötter durchaus geläufig, nicht jedoch als Gattungsbezeichnung für Schmähschriften (libels). ${ }^{55}$ Inwieweit das mit

52 Kuhn (2010), Ballads, hier S. 1623f. Anders in seiner Bamberger Habilitationsschrift Die Politik des Pasquino. Schmähschriften, Protestgelächter und Öffentlichkeiten in politischen Konflikten Alteuropas (ca. 1540-1750) von 2015, die bislang unveröffentlicht ist.

53 Buehler (2015) Defamation, S. 54.

54 So Dingemanse/Meijer Drees (2006) Pasquino, S. $479 f$. in einer Seitenbemerkung, bevor sie sich der literarischen Tradition als ihrem Hauptgegenstand zuwenden.

55 Fox (2000) Culture, S. 320. Allgemein waren im englischen Sprachraum, libels' ganz im Sinne von Gottsched und der unten diskutierten rechtlichen Tradition des, libellus famosus' definiert ("Genuine libels in verse or prose anderen Genrekonventionen auf der Insel zu tun hatte, ist eine der vielen offenen Fragen. Libels hatten dort oft die Form von Balladen, die auf populäre Melodien gesungen wurden und so weiter Verbreitung fanden. Oft wurden sie gemeinschaftlich beim Trunk in der Taverne gedichtet. Wer nicht schreiben konnte, fragte Reisende um Mithilfe, und gelegentlich bediente man sich sogar für die Aufführung professioneller Balladensänger. ${ }^{56}$ Allerdings war das Dichten und Singen spöttischer Lieder auch in Deutschland keineswegs unbekannt. ${ }^{57}$ Insgesamt bedürfte die Frage, in welchen Regionen Europas der Begriff ,Pasquill' eine handschriftliche Schmähschrift bezeichnete, einer genaueren Recherche.

Diese Feststellung trifft, drittens, auch auf die zeitliche Dimension zu, auf die Frage nämlich, ab wann ein solcher Begriffsgebrauch im deutschsprachigen Raum beobachtet werden kann. Einen gewissen Terminus post quem markiert die zitierte Reichspolizeiordnung von 1548 mit ihrem Verbot ,pasquillischer' Texte; sie indiziert, dass der Begriff zu dieser Zeit im Schwange war, nicht jedoch, dass er, im weiten Sinn, als Synonym für Schmähschrift benutzt wurde. Der Titel des 1552 kompilierten, in insgesamt drei Exemplaren erhaltenen Herbrotbuchs (Paßquillus Dialogus) ${ }^{58}$, in dem zahlreiche Schmähschriften über den Augsburger Bürgermeister Jacob Herbrot, einen reichen sozialen Aufsteiger, gesammelt sind, indiziert eine gewisse Popularität des Begriffs in der Stadt am Lech. Ob es sich um eine Sammlung tatsächlich in der Stadt verteilter Pasquille handelt, erscheint zumindest fragwürdig. ${ }^{59}$

are personally malicious, designed to humiliate specific victims", May/Bryson [2016] Libel, S. 1). Von diesen heimlichen Angriffen konnten dann, pasquils' im Sinne einer allgemeinen, offen vorgetragenen und literarisch stilisierten Satire unterschieden werden (Croft [1995] Libels, S. 267). 56 Fox (2000) Culture, S. 299-334, der sich u.a. auf ein breites Sample von Gerichtsfällen (Star Chamber) stützen kann, vgl. ebd., S. 309.

57 So etwa in Augsburg, wo 1579 der Pflasterer Sebastian Hundertkass aus der Stadt verbannt wurde, weil er verwegene Balladen gedichtet und handschriftlich an sein Umfeld verteilt hatte (Creasman [2012] Censorship, S. 29f.). Vgl. für einen regelrechten Sängerkrieg zwischen Nachbarn unterschiedlicher Konfession im Jahr 1601 ebd., S. $132 \mathrm{f}$.

58 HAB Cod. Guelf. 80.4 Aug $2^{\circ}$.

59 Vgl. vorerst Kuhn (2007) Laughter. Eine umfassendere Auswertung findet sich in seiner Habilitationsschrift, vgl. oben Fn. 52. 
Ein Kölner Edikt vom Dezember 1555 verbietet wiederum "allerlei Schmehegedichte, die man Pasquillen nennet" und die auf bestimmte, geistliche oder weltliche Standespersonen "erdichtet" würden. ${ }^{60}$ Der Kontext macht deutlich, dass es sich nicht um literarische, gedruckte Werke handelte, sondern wohl eher um ad hoc entstandene handschriftliche Texte, die offenbar in Reimform abgefasst waren. Das trifft ebenfalls auf eine anonym verbreitete Herrn Niemands Predigt zu, die 1566 in gelehrten Kreisen Nordsachsens kursierte und die heftige Anwürfe gegen den Kurfürsten August enthielt. In den Untersuchungsakten wird die Schrift nicht nur als "famos libell", sondern einige Mal auch als "pasquil" etikettiert. ${ }^{61}$ Im bikonfessionellen Augsburg des späteren 16 . Jahrhunderts waren Pasquille als Medium der konfessionellen Auseinandersetzung dann durchaus gängig, etwa als 1584 im Zuge des Kalenderstreites im Weberzunfthaus und an anderen Orten derartige Texte ausgelegt wurden; vergeblich versuchte der Rat damals, Verdächtige mittels Schreibproben zu überführen. ${ }^{62}$ Diese wenigen, eher episodischen Belege vermögen keine systematische (freilich sehr aufwändige) Recherche zu ersetzen. Sie belegen aber mit hinreichender Klarheit, dass sich im Laufe der zweiten Hälfte des 16. Jahrhunderts in verschiedenen Regionen des Reiches der Sprachgebrauch vom Pasquill als handschriftlicher, anonymer Schmähschrift etabliert hatte. Ganz selbstverständlich drohen die Hamburger Statuten von 1605 den Verfassern von Schmähschriften und "Paßquillos" Gefängnis oder Verbannung an. ${ }^{63}$ Ein entsprechender Sprachgebrauch sollte bis weit in die Sattelzeit um 1800 geläufig bleiben. ${ }^{64}$

60 HAStK Bestand 114, Edikte 13, Nr. 112. Nach Schmidt (1985), Libelli Famosi, S. 281, appelliert Herzog Christoph von Württemberg im Jahr 1557 an die Buchdrucker, keine "Invektiven, Pasquille oder andere Schmach-, Schandoder sonst andere Schriften, so Unruhe anrichten möchten", ausgehen zu lassen.

61 HStAD, 10024 Geheimer Rat (Geheimes Archiv), Loc. 9667/9, fol. 18v. u.ö. Vgl. Rose (2020) Schmähschriften.

62 Creasman (2012) Censorship, S. 165. Vgl. auch Roeck (1989), Stadt, Stichwort ,Pasquille, Schmähschriften' im Sachregister.

63 Müller (2017) Verletzende Worte, S. 80, Fn. 166.

64 Die Datierung auch bei Gestrich (1997) Schandzettel, S. 45; reichhaltiges Material bei Rublack (1995) Anschläge; Bellingradt (2011) Flugpublizistik.
Trifft die Beobachtung zu, dass es in der zweiten Hälfte des 16 . Jahrhunderts zu einer räumlichen Diffusion des Etiketts ,Pasquill' im deutschen Sprachraum gekommen ist, dann könnte damit zugleich eine Veränderung in Bezug auf das Gattungsformat verbunden gewesen sein. In der Frühzeit scheint zur herabsetzenden, spöttischen bzw. ehrverletzenden Qualität der Schmähschrift noch eine gewisse künstlerisch-satirische Ambition (etwa in Gestalt von Reimen) hinzutreten zu müssen, damit die Zeitgenossen von einem ,Pasquill sprachen. Etwas zugespitzt formuliert: Für sie mochten zwar alle Pasquille den Charakter von Schmähschriften haben, aber weitaus nicht alle Schmähschriften wären aufgrund ihrer formalen Erscheinungsform von ihnen als Pasquill betrachtet worden. ${ }^{65}$ Nach und nach verlor sich dann wohl dieses spezifische Verständnis gänzlich, wie die eingangs angeführten Zitate belegen.

\section{Zwischen Schmähung und Kritik}

Der Begriff Schmähschrift beinhaltet bis heute die abwertende Etikettierung einer Sprachhandlung. Libelli famosi wurden im juristischen Diskurs als Anschlag auf die persönliche Ehre und als Gefahr für die öffentliche Ordnung negativ bewertet. Allerdings gab es bereits im Rechtsdenken Ansatzpunkte dafür, diese Sicht der Dinge zu relativieren und zu differenzieren. Falls die Wahrheit der vorgebrachten Schmähung bewiesen werden könnte und falls dieser Wahrheitsbeweis dem Gemeinen Nutzen diente, so die Meinung einiger Juristen, dann verkehrte sich eine ehrmindernde Schmähung in die lobenswerte Anzeige sträflichen Fehlverhaltens. In diesem Fall, so z. B. der sächsische Rechtsgelehrte König in seinem Handbuch, solle der Verfasser "nicht alleine vngestrafft bleiben/ sondern sol auch gelobet vnd begabet werden". ${ }^{66}$

Die Karriere des Begriffs, ,Pasquill' als Synonym für Schmähschrift unterstreicht die sich hier andeutende Ambivalenz der Bewertung noch ein-

65 Ich verdanke diese Beobachtung Jan Siegemund. 66 König (1541) Processus, Bl. 66vf. Vgl. zum Hintergrund Bartels (1959) Dogmatik, S. 171-207; Müller (2017) Verletzende Worte, S. 113; Vgl. jetzt für einen Fall, bei dem der Charakter der inkriminierten Schriften und der animus iniurandi des Täters strittig war, Siegemund (2020) Schmähschriftenprozess. 
mal nachdrücklich. Mit der römischen Figur des Pasquino verband sich ursprünglich eine Lizenz zum Spott gegenüber und zur Kritik an den Mächtigen, eine Lizenz, die in der italienischen Literatur noch lange erhalten blieb. ${ }^{67}$ Die Überblendung der mitteleuropäischen Schmähschriften mit dieser Pasquino-Tradition kann mithin als Indiz für eine unterschwellige Aufwertung dieser Kommunikationsform als Medium der Kritik verstanden werden. Aber auch umgekehrt ist diese Entwicklung lesbar, als obrigkeitlicher Versuch, mit Blick auf die Gefahren für die eigene Autorität und für die öffentliche Ordnung die Lizenz zu delegitimieren und das kritische Potential des anonymen Spotts durch Kriminalisierung als ,Schmähschrift' zu unterdrücken.

Gleich, wie diese gegenläufigen Tendenzen bei intensiverer Erforschung zu gewichten sein werden, erforderlich erscheint schon jetzt eine Revision gegenüber der Hauptlinie der aufklärerischen Bewertung, wie sie von Gottsched repräsentiert wird und seither auch die einschlägige Forschung dominiert: Das Pasquill kann nicht als Gegenpol zur Kritik verstanden werden, sondern es steht vielmehr mitten im Schnittfeld von persönlicher Schmähung und sachlicher Kritik. Dabei war das Mischungsverhältnis zwischen beiden je nach Fall höchst unterschiedlich gelagert. Die PasquillSammlung gegen den ehemaligen Bürgermeister Jacob Herbrot in Augsburg stigmatisierten inn persönlich als konfessionellen, ökonomischen und moralischen Abweichler. Die drohenden Schmähschriften gegen die Kölner Bürgermeister im Gefolge des Gülich-Aufstandes richteten sich dagegen nicht gegen einen bestimmten Herrschaftsträger, sondern gegen die führenden Repräsentanten des politischen Systems. In höchst aggressiver Weise beschimpfte die Predigt des Herrn Niemand in Sachsen 1566 den Kurfürsten August als üblen Schinder von Land und Leuten und richtete sich gegen sein Steuerregime ebenso wie gegen seine Jagdleidenschaft; persönliche Schmähung und politische Kritik gingen hier eine extreme Mischung ein. ${ }^{68}$ Alle diese

67 Vgl. für das frühe 17. Jahrhundert z.B. Müller (im Erscheinen) Maler.

$68 \mathrm{Zu}$ Herbrot oben Anm. 59, zu Köln oben Anm. 17. Der Wortlaut der sächsischen Predigt in HStAD, 10024 Geheimer Rat (Geheimes Archiv), Loc. 9667/9, fol. 141r-146v. Vgl. Rose (2020) Schmähschriften.
Fälle besaßen gleichsam ein systemisches Element. Natürlich gab es auch solche Pasquille, die sich nicht gegen Herrschaftsträger richteten, sondern gegen andere Privatleute. Meist sind derartige Fälle, wie etwa die Schmähschriftenkampagne des Andreas Langner in Dresden 1569, durch ein deutlich asymmetrisches Machtverhältnis gekennzeichnet: Anders als durch öffentlichen Betrugs- und Beleidigungsvorwürfe glaubte Langner seinen Kontrahenten, den kurfürstlichen Lehnsmann Tham Pflugk, nicht auf den Rechtsweg zwingen zu können. ${ }^{69}$ Zumindest handelte es sich beim Pasquill also sehr häufig um ein typisches Instrument in der Hand von Machtunterlegenen; waren Herrschafts- und Amtsträger beteiligt, konnte ihr Einsatz sogar eine regelrechte ,Gegenöffentlichkeit' konstituieren, die fallweise das Potential besaß, das Meinungsspektrum zu erweitern oder sogar alternative Entscheidungen zu erzwingen. ${ }^{70}$

\section{Resümee}

Das Pasquill war - nimmt man seine heterogenen Erscheinungsformen zusammen - in keinerlei Hinsicht ein klar abgrenzbares Genre, weder im Sinn einer literarischen (konventionalisierten Textgruppe mit klaren Formenmerkmalen $)^{71}$ noch im Sinn einer kommunikativen Gattung (verfestigte Lösungsmuster kommunikativer Probleme $)^{72}$. Ebenso wenig präzise lässt sich die mediale Erscheinungsform des Pasquills erfassen. Selbst die Anonymität des Verfassers ist kein Ausschlusskriterium. Charakterisieren lässt sich das Pasquill bei aller Unschärfe vielleicht am besten durch die Tatsache, dass inm ein gattungsübergreifender Kommunikationsstil eingeschrieben scheint, ein invektiver Kommunikationsmodus. ${ }^{73}$ Dabei verband sich mit dem Etikett Pasquill die Vorstellung, dass es sich um eine Schmähung ad hominem handele, dass also persönliche Eigenschaften und Schwächen konkreter Personen an den Pranger gestellt werden. Keineswegs aber

69 Siegemund (2020) Schmähschriftenprozess. 70 Schwerhoff (2004) Öffentliche Räume, S. 126-133. 71 Hempfer (2007) Gattung.

72 Ауаß (2011) Kommunikative Gattungen, S. 278. 73 Vgl. Ellerbrock/Koch/Müller-Mall et al. (2017) Invektivität; Schwerhoff (2020) Invektivität. 
bedeutet das zwingend, dass sich mit dieser persönlichen Herabsetzung keine sachliche, auf allgemeine politische und gesellschaftliche Tatbestände zielende Kritik verbinden konnte. Vielleicht verdienen die Pasquille sogar umgekehrt einen prominenteren Platz in der Entwicklungsgeschichte einer öffentlichen Sphäre der Kritik, in der lange Zeit die persönliche und die sachliche Ebene kaum voneinander zu trennen waren. Kritik an den konkreten Verhältnissen war regelmäßig verknüpft mit Kritik an konkreten Herrschaftsträgern. Es ist deshalb keine Übertreibung, wenn Pasquille - obwohl sie durchaus oft sehr privaten Interessen dienten - in der Literatur als ein zentrales Medium des Protestes gegen und der Kritik an der Obrigkeit firmieren. ${ }^{74}$

Die Trennung von persönlicher Schmähschrift und sachlicher Kritik durch den Aufklärer Gottsched, mit dem dieser Beitrag eingeleitet wurde, darf deshalb nicht als Beschreibung der Tatsachen missverstanden werden, sondern als ein normatives Postulat mit ganz eigener invektiver Qualität. Es repräsentierte durchaus die Hauptströmung aufklärerischer Haltung zur Frage der Pasquille, unbeschadet der Tatsache, dass manche doch etwas differenzierter argumentierten. Das war etwa der Fall bei jener 1783 erschienenen Verteidigung der Pasquille, die zwischen verwerflichen Schmähschriften als individuellen Racheaktionen einerseits und "vernünftigen Pasquillanten", die mit ihren Schriften moralische Fehler aufdeckten, die für das gesamte Publikum schädlich wären, andererseits unterschieden sehen wollte. ${ }^{75}$ Und auch Carl Friedrich Bahrdt war 1787 die Abwägung zwischen Presse- und Meinungsfreiheit und den jeweiligen Persönlichkeitsrechten bzw. dem individuellen Ehrschutz eine lange Abhandlung wert. $^{76}$ Der Theologe und Schriftsteller Bahrdt war für diese Aufgabe durchaus prädestiniert, denn er selbst war sowohl ein verurteilter Pasquillant wie die Zielscheibe einer Schmähschrift: Das 1790 gedruckte Schauspiel Doctor Bahrdt mit der eisernen Stirn zeichnete in vier Aufzügen ein drastisches Bild vom körperlichen Verfall und der sittlichen Entartung der Crème de la Crème der norddeutschen Spätaufklärung, angefangen

74 Rublack (1995) Anschläge, S. 409; Gestrich (1997) Schandzettel, S. 55.

75 Nach Grunert (2019) Streiten und Strafen, S. $186 f$.

76 Grunert (2019) Streiten und Strafen, S.187f. mit der Titelfigur jenes Doktors mit der eisernen Stirn, von der permanent der giftgrüne Angstschweiß triefte. Das Bahrdt-Pasquill, als dessen Verfasser sich später der Theaterschriftsteller August von Kotzebue bekannte, sollte für weitläufige gerichtliche und literarische Auseinandersetzungen sorgen. ${ }^{77}$ Es stellt ein sicherlich extremes, aber keineswegs vollkommen solitäres Exempel dafür dar, wie wenig die Gottsched'sche Trennung zwischen Schmähung und Kritik selbst in der Hochzeit der Aufklärung der Wirklichkeit entsprach.

Wie der Blick in die Rechtshandbücher und die archivalische Überlieferung zeigt (und wie weitere Forschungen erhärten müssten), war die hauptsächliche Erscheinungsform des frühneuzeitlichen Pasquills im deutschen Sprachraum nicht das literarisch gedrechselte Druckerzeugnis, sondern der situativ platzierte, oft mehr oder weniger improvisierte handschriftliche Zettel, dessen Text gereimt sein konnte, aber nicht musste, und der außerdem durch Bilder ergänzt sein konnte. Der geringe Aufwand bei der Produktion und das relativ kalkulierbare Risiko machten das Pasquill zur klassischen, weapon of the weak' (Scott) im Zeitalter der Staatsbildung. Anonymität und Einsatz in solchen Situationen, die von einem großen politischen bzw. sozialen Machtgefälle gekennzeichnet waren, vervollständigen das Profil des Pasquills i.e.S.: Insofern scheint seine Charakterisierung als ein spezifisches Kommunikationsmedium zulässig, das sich durch die besondere Verbindung von Handschriftlichkeit, Anonymität, öffentlicher Platzierung und invektiver Adressierung auszeichnet.

Die Schlichtheit dieses Pasquills sollte nicht dazu verleiten, darin ein altmodisches, gleichsam nicht mehr auf der Höhe der Zeit befindliches Mittel der Kommunikation zu sehen. Dagegen spricht nicht allein die erhöhte Sensibilität der Obrigkeiten seit dem Eintritt in das Druckzeitalter, sondern auch die fortdauernde Bedeutung einer Face-to-Face - Kommunikation, wo sachliche Kontroversen eng mit der Bedrohung persönlicher Ehre verknüpft waren. ${ }^{78}$ Das Pasquill erweist sich als durchaus kongenial zu einer frühneuzeitlichen Anwesenheitsgesellschaft, die noch sehr stark durch Mündlichkeit geprägt war;

77 Erkner/Siebers (2004) Das Bahrdt-Pasquill.

78 Schwerhoff (2004) Öffentliche Räume, S. 133-136. 
einer Gesellschaft zugleich, deren Bewusstsein für die neuen Möglichkeiten bzw. - je nach Perspektive - neuen Gefahren von Öffentlichkeit im Zeitalter der Druckmedien zunehmend geschärft war. ${ }^{79}$ Die handschriftlichen libelli hatten ein groBes Wirkungspotential in einem frühneuzeitlichen Mediensystem, in dem mündlich weitergetragene Gerüchte, handschriftliche Zettel und gedruckte Pamphlete komplex zusammenwirkten. Neben den Druckerzeugnissen prägten sie in charakteristischer Weise die frühneuzeitliche Öffentlichkeit, indem sich in innen persönliche Herabsetzung und sachliche Kritik eng verschränkten..$^{80}$ Bei ihrer weiteren Erforschung empfiehlt es sich für alle Forschenden, nicht durch die aufklärerische Brille eines Johann Christoph Gottsched auf das Phänomen zu schauen.

\section{Literaturverzeichnis}

\section{Quellen}

Anonym (um 1520): Pascuillus Ain warhafftiges büchlein Erklerend was list die Römer brauchen, mit Creiren viler Cardinäl, auff das sy alle Bistumb Deijtscher Land vnder sich bringen. Augsburg (VD16 P 850).

Anonym (1537): Unterredung / zwischen dem Pasquillen / und Deudschen / von dem zukünfftigen Concilio zu Mantua [...]. Wittenberg (VD16 U 188).

Anonym (1546): Pasquillus Germanicus In Quo Causa praesentis belli attingitur. o. O. (VD16 P 843).

Anonym (1546): Pasquillus. Newe Zeytung Vom Teüffel: Wie newlich der Bapst vnd sein Gesell, Der oberst Sathanas auß der Hell, Von jrer gsellschafft vnd disem Kryg, Sprach gehalten, vnd wem sy den Syg, All baid hertzlich gern günnen wollten [...]. Augsburg (VD16 S 3175).

Eckhardt, Karl August (1974): Schwabenspiegel Kurzform I. Landrecht II. Lehnrecht (MGH Fontes iuris Germanici antiqui N. S. IV 1-2), zweite, neubearbeitete Auflage. Hannover: Hahn.

Gottsched, Joh. Christoph (1758): Beobachtungen über den Gebrauch und Mißbrauch vieler deutscher Wörter und Redensarten. Straßburg/ Leipzig: Johann Amandus König.

Hauptstaatsarchiv Dresden (HStAD), 10024 Geheimer Rat (Geheimes Archiv), Loc. 9667/9.

Haus-, Hof- und Staatsarchiv (HHStA) Wien, Bestand Reichskanzlei, Diplomatische Akten, Köln: Berichte 2a.

79 Vgl. Schlögl (2014) Anwesende und Abwesende.

80 Vgl. exemplarisch Bellingradt (2009) "Lateinische Zeddel"; Sennefelt (2008) Citizenship; jetzt Beckert/Kästner/ Schwerhoff et al. (2020) Invektive Kommunikation und Öffentlichkeit.
Herzog August Bibliothek Wolfenbüttel (HAB), Cod. Guelf. 80.4 Aug 2०: Paßquillus Dialogus/ Tragedia/ Supplicationes/ Reimen/ Testament/ Lieder/ Urkunden/ Beschreybungen/ Quittungen/ Schadloßbrieff/ Edicta/ Citation/ sambt den Abschiden/ unnd andern mehr schrifften, so zu Newburg aufgelegt worden/ Den Jacob Herbrot/ geweßnen Burgermaister In Augspurg belangend/ so wider Ine über seine böse unnd schändtliche geübte thatten gemacht unnd offenbar worden... 1552 (online unter: http://diglib.hab.de/ mss/80-4-aug-2f/start.htm, 3.1.2020).

Historisches Archiv der Stadt Köln (HAStK), Bestand 35, Verfassung und Verwaltung G 228.

Historisches Archiv der Stadt Köln (HAStK), Bestand 114, Edikte 13.

König, Kilian (1541): Processus und Practica der Gerichtsleuffte / Nach sechsischem gebrauch [...]. o.O.

Senckenberg, Heinrich Chr. von (1747): Neue und vollständigere Sammlung der Reichs-Abschiede [...] In Vier Theilen. Frankfurt am Mayn: Koch.

\section{Forschungsliteratur}

Ayaß, Ruth (2011): Kommunikative Gattungen, mediale Gattungen. In: Habscheid, Stephan (Hg.): Textsorten, Handlungsmuster, Oberflächen. Linguistische Typologien der Kommunikation. Berlin/ New York: De Gruyter, S. 275-295.

Bartels, Karlheinz (1959): Die Dogmatik der Ehrverletzung in der Wissenschaft des Gemeinen Rechts bis zum Ausgang des 18. Jahrhunderts. Diss. jur. Göttingen.

Bauer, Oswald (2008): Pasquille in den Fuggerzeitungen. Spott- und Schmähgedichte zwischen Polemik und Kritik (1568-1605). Wien/Köln/Weimar: Böhlau.

Beckert, Stefan/Kästner, Alexander/Schwerhoff, Gerd et al. (2020): Invektive Kommunikation und Öffentlichkeit - Konstellationsanalysen im 16. Jahrhundert. In: Jahrbuch für Kommunikationsgeschichte 22, S. 36-82.

Bellingradt, Daniel (2011): Flugpublizistik und Öffentlichkeit um 1700. Dynamiken, Akteure und Strukturen im urbanen Raum des Alten Reiches. Stuttgart: Steiner.

Bellingradt, Daniel (2009): „Lateinische Zeddel" in der Reichsstadt Köln (1708). Signale, Diskurse und Dynamiken im öffentlichen urbanen Raum der Frühen Neuzeit. In: Geschichte in Köln 56, S. 207-237.

Buehler, Paul (2015): "So that the Common Man may see what kind of tree bears such harmful fruit": Defamation, Dissent, and Cencorship in the Holy Roman Empire, ca. 1555-1648, Diss. University of Arizona. URL: https://repository.arizona.edu/ handle/10150/581330?show=full (letzter Zugriff: 20.11.2020).

Burke, Peter (1986): Beleidigungen und Gotteslästerung im frühneuzeitlichen Italien. In: Ders.: Städtische Kultur in Italien zwischen Hochrenaissance und Barock. Eine historische Anthropologie. Berlin: Wagenbach, S. 96-110, $205 f$. 
Creasman, Allyson F. (2012): Censorship and Civic Order in Reformation Germany, 1517-1648. Aldershot/ Burlington: Ashgate.

Croft, Pauline (1995): Libels, Popular Literacy and Public Opinion in Early Modern England. In: Historical Research 68, S. 266-285.

Damianaki, Chrysa/Procaccioli, Paolo/Romano, Angelo (Hgg.) (2006): Ex Marmore. Pasquini, Pasquinisti, Pasquinae nell'Europa Moderna. Atti del Colloquio internationale Lecce-Otranto, 17-19 novembre 2005. Manziana: Vecchiarelli.

Dingemanse, Clazina/Meijer Drees, Marijke (2006): Pasquino in Early Modern Dutch Pamphlet Literature (ca. 1500-1750). In: Damianaki, Chrysa/Procacciolo, Paolo/Romano, Angelo (Hgg.): Ex Marmore. Pasquini, Pasquinisti, Pasquinae nell'Europa Moderna. Atti del Colloquio internationale Lecce-Otranto, 17-19 novembre 2005. Manziana: Vecchiarelli, S. 477-498.

Eintrag ,Pasquino'. Wikipedia: https://de.wikipedia.org/w/ index.php?title=Pasquino\&oldid=186209496 (letzter Zugriff: 02.01.2020).

Ellerbrock, Dagmar/Koch, Lars/Müller-Mall, Sabine et al. (2017): Invektivität - Perspektiven eines neuen Forschungsprogramms in den Kultur- und Sozialwissenschaften. In: Kulturwissenschaftliche Zeitschrift 2/1, S. 2-24. URL: https://doi.org/10.2478/ kwg-2017-0001 (letzter Zugriff: 29.03.2020).

Erkner, Brigitte/Siebers, Winfried (2004): Das BahrdtPasquill. Ein publizistischer Streit zwischen Aufklärung und Gegenaufklärung 1790-1796. In: Goldenbaum, Ursula (Hg.): Appell an das Publikum. Die öffentliche Debatte in der deutschen Aufklärung 1687-1796. Berlin: De Gruyter, S. 897-939

Fenster, Thelma/ Smail, Daniel (Hgg.) (2003): Fama. The Politics of Talk and Reputation in Medieval Europe. Ithaca/London: Cornell University Press.

Fox, Adam (2000): Oral and Literature Culture in England, 1500-1700. Oxford: University Press.

Fuchs, Ralf-Peter (1999): Um die Ehre. Westfälische Beleidigungsprozesse vor dem Reichskammergericht 1525-1805. Paderborn: Ferdinand Schöningh.

Gestrich, Andreas (1997): Schandzettel gegen die Obrigkeit. Pasquillen als Mittel der Herrschaftskritik im Ancien Régime. In: Borst, Otto (Hg.): Südwestdeutschland. Die Wiege der deutschen Demokratie. Tübingen: Silberburg, S. 43-57, 206-208.

Goldenbaum, Ursula (Hg.) (2004): Appell an das Publikum. Die öffentliche Debatte in der deutschen Aufklärung 1687-1796. Berlin: De Gruyter.

Grunert, Frank (2019): Streiten und Strafen. Der libellus famosus als Tatbestand des Strafrechts und als Streitmedium. In: Zeitschrift für Neuere Rechtsgeschichte 41, S. 173-196.

Haug-Moritz, Gabriele (2002): "Geschwinde Welt". Krieg und öffentliche Kommunikation - zur Erfahrung beschleunigten historischen Wandels im Heiligen Römischen Reich deutscher Nation in der ersten Hälfte des 16. Jahrhunderts (1542-1554). In: Militär und Gesellschaft in der frühen Neuzeit 6, S. 139-148.
Hempfer, Klaus W. (2007): Art. ,Gattung'. In: Reallexikon der deutschen Literaturwissenschaft Bd. 1. Berlin: De Gruyter, S. 651-655.

Historisches Archiv der Stadt Köln (1996): Stadtrat, Stadtrecht, Bürgerfreiheit. Ausstellung aus Anlass des 600. Jahrestages des Verbundbriefes vom 14. September 1396. Köln: Historisches Archiv.

Holenstein, Pia/Norbert Schindler (1992): Geschwätzgeschichte(n). Ein kulturhistorisches Plädoyer für die Rehabilitierung der unkontrollierten Rede. In: Dülmen, Richard van (Hg.): Dynamik der Tradition. Frankfurt am Main: Fischer, S. 41-108.

Hess, Günter (2003): Pasquill. In: Reallexikon der deutschen Literaturwissenschaft Bd. 3. Berlin: De Gruyter, S. 31-34.

Kaufmann, Thomas (2006): Konfession und Kultur. Lutherischer Protestantismus in der zweiten Hälfte des Reformationsjahrhunderts, Tübingen: Mohr Siebeck.

Kuhn, Christian (2010): Ballads, Libels, and Songs. In: Classen, Albrecht (Hg.): Handbook of Medieval Studies. Terms - Methods - Trends. Berlin/New York: De Gruyter, S. 1618-1633.

Kuhn, Christian (2007): Urban Laughter as a 'CounterPublic' in early modern Augsburg: The case of the City Mayor, Jakob Herbrot (1490/95-1564), In: t'Hart, Marjolein/Bos, Dennis (Hgg.): Humour and Social Protest. Cambridge: University Press, S. 77-93.

Lastraioli, Chiara (2003): Les „Pasquini" - un „lieu de mémoire" européen? In: Schmale, Wolfgang ( $\mathrm{Hg}$.$) :$ Kulturtransfer. Kulturelle Praxis im 16. Jahrhundert. Wien: Studienverlag, S. 461-475.

Lastraioli, Chiara (2006): Pasquillus exul: note sulla diffusione di testi e temi pasquineschi al di là delle Alpi. In: Damianaki, Chrysa/Procacciolo, Paolo/ Romano, Angelo (Hgg.): Ex Marmore. Pasquini, Pasquinisti, Pasquinae nell'Europa Moderna. Atti del Colloquio internationale Lecce-Otranto, 17-19 novembre 2005. Manziana: Vecchiarelli, S. 429-475.

Lastraioli, Chiara (2010): Pétromarchies renaissantes: de la dispute rituelle au combat des idées à I'ombre des ,Pierres parlantes'. In: Laureys, Marc/ Simons, Roswitha (Hgg.): Die Kunst des Streitens. Inszenierung, Formen und Funktionen öffentlichen Streits in historischer Perspektive. Göttingen: V\&R unipress, S. 219-243.

Lentz, Matthias (2004): Konflikt, Ehre, Ordnung. Untersuchungen zu den Schmähbriefen und Schandbildern des späten Mittelalters und der frühen Neuzeit (ca. 1350 bis 1600). Mit einem illustrierten Katalog der Überlieferung. Hannover: Hahn.

Matthias, E. (1888): Ein Pasquill aus der Zeit des Schmalkaldischen Krieges. In: Zeitschrift für deutsche Philologie 20, S. 151-188.

May, Steven/Bryson, Alan (2016): Verse Libel in Renaissance England and Scotland. Oxford: University Press.

Müller, Jürgen (2021): Der Maler als Pasquino - Spott, Kritik und Subversion. Eine neue Deutung von Caravaggios Amor vincitore. In: Israel, Uwe/Kraus, 
Marius/Sasso, Ludovica (Hgg.): Agonale Invektivität. Konstellationen und Dynamiken der Herabsetzung im deutschen und italienischen Humanismus. Berlin: De Gruyter.

Müller, Mario (2017): Verletzende Worte. Beleidigung und Verleumdung in Rechtstexten aus dem Mittelalter und aus dem 16. Jahrhundert. Hildesheim: Universitätsverlag.

Oesterle, Günter (1986): Das „Unmanierliche” der Streitschrift. Zum Verhältnis von Polemik und Kritik in Aufklärung und Romantik. In: Worstbrock, Franz Josef/Koopmann, Helmut (Hgg.): Formen und Formgeschichte des Streitens. Der Literaturstreit. Tübingen: Niemeyer, S. 107-120.

Reynolds, Anne (1985): Cardinal Oliviero Carafa and the early cinquecento tradition of the feast of Pasquino. In: Humanistica Lovaniensia. Journal of Neo-Latin Studies 34a, S. 178-208.

Roeck, Bernd (1989): Eine Stadt in Krieg und Frieden. Studien zur Geschichte der Reichsstadt Augsburg zwischen Kalenderstreit und Parität. Göttingen: Vandenhoeck \& Ruprecht.

Romano, Angelo (2006): La satira di Pasquino: formazione di un genere letterario. In: Damianaki, Chrysa/Procacciolo, Paolo/Romano, Angelo (Hgg.): Ex Marmore. Pasquini, Pasquinisti, Pasquinae nell'Europa Moderna. Atti del Colloquio internationale Lecce-Otranto, 17-19 novembre 2005. Manziana: Vecchiarelli, S. 11-34.

Rose, Max (2020): Schmähschriften gegen Kurfürst August von Sachsen. Wertungen - Kontexte - Öffentlichkeit(en). Masterarbeit im Fach Geschichte an der TU Dresden.

Rublack, Ulinka (1995): Anschläge auf die Ehre. Schmähschriften und -Zeichen in der städtischen Kultur des Ancien Régime. In: Schreiner, Klaus/Schwerhoff, Gerd (Hgg.): Verletzte Ehre. Ehrkonflikte in Gesellschaften des Mittelalters und der Frühen Neuzeit. Köln et al.: Böhlau, S. 381-411.

Schade, Oskar (1863): Satiren und Pasquille aus der Reformationszeit. 3 Bände. Hannover: Rümpler.
Schlögl, Rudolf (2014): Anwesende und Abwesende. Grundriss für eine Gesellschaftsgeschichte der Frühen Neuzeit. Konstanz: University Press.

Schmidt, Günther (1985): Libelli Famosi. Zur Bedeutung der Schmähschriften, Scheltbriefe, Schandgemälde und Pasquille in der deutschen Rechtsgeschichte. Diss. Masch. Köln.

Schwerhoff, Gerd (2004): Öffentliche Räume und politische Kultur in der frühneuzeitlichen Stadt: Eine Skizze am Beispiel der Reichsstadt Köln. In: Schlögl, Rudolf (Hg.): Interaktion und Herrschaft. Die Politik der frühneuzeitlichen Stadt. Konstanz: UVK, S. 113-136.

Schwerhoff, Gerd (2017): Köln im Ancien Régime 1686 1794. Köln: Greven.

Schwerhoff, Gerd (2020): Invektivität und Geschichtswissenschaft. Konstellationen der Herabsetzung in historischer Perspektive - ein Forschungskonzept. In: Historische Zeitschrift 311, S.1-36

Sennefelt, Karin (2008): Citizenship and the Political landscape of Libelling in Stockholm, c. 1720-70. In: Social History 33/2, S. 145-163.

Siegemund, Jan (2020): unrechtliche peinliche schmehung oder dem gemeinen nutz nuetzlich? Eine Fallstudie zur Normenkonkurrenz im Schmähschriftprozess des 16. Jahrhunderts. In: Das Mittelalter 25/1, S. $135-149$.

Voigt, Johannes (1838): Über Pasquille, Spottlieder und Schmähschriften aus der ersten Hälfte des sechzehnten Jahrhunderts. In: Raumer, Friedrich von (Hg.): Historisches Taschenbuch Bd. 9. Leipzig: Brockhaus, S. 321-524.

Waldeck, Oskar (1910/11): Die Publizistik des Schmalkaldischen Krieges. In: Archiv für Reformationsgeschichte 8, S. 44-133 [Teil I ebd. 7 (1909/10), S. 1-55].

Wolf, Thomas (2003): Pasquill. In: Ueding, Gert et al. (Hgg.): Historisches Wörterbuch der Rhetorik, Bd. 6. Berlin: De Gruyter, Sp. 682-686. 